\title{
Perioperative quantitative coronary analysis and ultrasonographic graft assessments for the right coronary artery bypass grafting
}

\author{
Shinya Takahashi", Keijiro Katayama, Taijiro Sueda \\ From World Society of Cardiothoracic Surgeons 25th Anniversary Congress, Edinburgh \\ Edinburgh, UK. 19-22 September 2015
}

\section{Background/Introduction}

Preoperative quantitative assessment of coronary angiography (CAG) and real-time graft size and graft flow assessment using epigraftic ultrasonography in the operative field have been performed to ascertain the patency in the right coronary artery bypass grafting (CABG).

\section{Aims/Objectives}

This study aims to evaluate the efficacy of the quantitative coronary artery and graft assessment to increase the patency of the right CABG.

\section{Method}

CABG was performed in 200 patients from January 2010 to December 2014. Ninety-three patients underwent all of preoperative CAG, real-time graft assessment and postoperative CAG with a total of 93 grafts anastomosed to the right coronary artery. Severity of coronary artery stenosis was evaluated by CAG. The size of target right coronary artery was measured by ultrasonography in the operative field. Parameters about graft flow were obtained from flow velocity curve in all the graft. The grafts were divided into two groups: patent grafts (Group A, $\mathrm{n}=86$ ) and failing grafts (Group B, $\mathrm{n}=7$ ). All factors were compared in these two groups and evaluated by logistic analysis and receiver operating characteristic (ROC) curve analysis.

\section{Results}

The overall patency as measured by postoperative CAG was $94.6 \%$ (88/93). There were 7 failing grafts including
5 occlusion and 2 competitive slow flow. Logistic regression analysis revealed that the percentage of graft size measured by ultrasonography divided by the size of the right coronary artery (graft-RCA size mismatch) and the pulsatility index (PI) were independent predictors of early graft failure (graft-RCA size mismatch, Odds ratio [OR], 2.37, 95\% confidence interval [95\%CI], 1.94-289.0, $\mathrm{p}=0.0132$; PI, OR, 1.51, 95\%CI, 1.16-1.96, $\mathrm{p}=0.0023$, area under the curve [AUC], 0.981). ROC curve analysis revealed that graft-RCA size mismatch $>2.08$ and PI > 4.55 were predictors of graft failure.

\section{Discussion/Conclusion}

In this series, epigraftic ultrasonography depicted graft flow clearly. Combination of preoperative quantitative coronary artery assessment and real time graft assessment was essential to predict graft failure during the CABG. This technique may increase the patency of the right coronary artery bypass grafting.

Published: 16 December 2015

doi:10.1186/1749-8090-10-S1-A200

Cite this article as: Takahashi et al:: Perioperative quantitative coronary analysis and ultrasonographic graft assessments for the right coronary artery bypass grafting. Journal of Cardiothoracic Surgery 2015 10(Suppl 1): A200. 